\title{
Antimalarial drug discovery: where next?
}

\author{
"This issue of Future Medicinal Chemistry brings together perspectives, reviews and preliminary communications from \\ leading international experts in the disease with considerable experience in drug hunting."
}

Malaria is a parasitic infection of the genus Plasmodium and has historically been one of mankind's deadliest diseases. The good news is that over the last decade there has been major progress in its control with 655,000 deaths reported by the WHO during 2010, compared with well over 1 million deaths at the turn of the millennium [1]. This reduction has been achieved thanks largely to the increased use of insecticide treated bed-nets, indoor residual spraying to kill mosquitos (the vector that carries the parasite and infects humans) and the availability and use of effective, safe and high-quality medicines [101].

The increased focus of the malaria community on eradication has arisen both in response to the moral imperative to save lives and eliminate the devastating disease burden, and also in recognition that a pure case-management approach is unsustainable. Thus renewed efforts are being made to develop insecticides that overcome known resistance pathways and kill all mosquitos, to deliver vaccines that protect infants and children and to discover, develop and deliver medicines that not only clear the asexual blood stage parasites, but also the asymptomatic forms (exo-eryothrocytic stages) that perpetuate the lifecycle of Plasmodium [2]. These asymptomatic stages include [3]:

- The sexual blood stage forms (gametocytes) that fertilise in the mosquito following a blood meal on a patient and ultimately render mosquitos infectious;

- The developing liver stage forms that ultimately lead to blood stage malaria after a period of around 6 days (in falciparum malaria);

- The dormant liver stages (hypnozoites) that exist in Plasmodium vivax and ovale and lead to relapsing blood stage malaria at a later time point, in the absence of a mosquito bite;

- The developing parasite stages in the mosquito (gametes, ookinetes, oocysts and sporozoites) that preserve the integrity of the parasite life cycle and ensure transmission.
It is clear that chemotherapy is still a vital component in the fight against malaria [4]. To protect new drugs from the risk of resistance, the WHO recommendation is that all treatments be combinations of two or more drugs such that no molecule is exposed as a monotherapy to high levels of parasites. Artemisinin combination therapies, the first-line medicines, are extremely safe and effective after three days of dosing. The next challenge posed by the Malaria Eradication Agenda has been to deliver combination products that can be given as a single dose to maximize compliance and reduce risk of resistance from under-dosing [5]. Given the emerging reports of resistance to artemisinin and other antimalarials, the need for new medicines is ever present.

Over the last decade there has been an increased investment in antimalarial $\mathrm{R} \& \mathrm{D}$ through the work of organizations such as Medicines for Malaria Venture (Geneva, Switzerland), their partners and others. New molecules with new modes of action are entering into preclinical development and beyond [6].

One area that is critical for success is the ability to understand and model the effects of antimalarials in preclinical models and in patients such that data from the former can predict the latter. The delivery of robust pharmacokinetic-pharmacodynamic models that link a drug exposure to its effect in a holistic manner will have a huge impact. It will help maximize the delivery of quality molecules by predicting human doses, and the delivery of quality medicines by optimizing resistance risks and combination partner choices.

When it comes to the strategy for finding new antimalarials there are two main approaches that are being adopted, both taking advantage of the incredible progress made in biotechnology in recent years. The first exploits the revolutions in biochemistry and genetics by using the sequencing of the parasite genomes combined with analyzing mechanisms and pathways to select relevant biological targets [7]. The second exploits the ability to miniaturize and culture

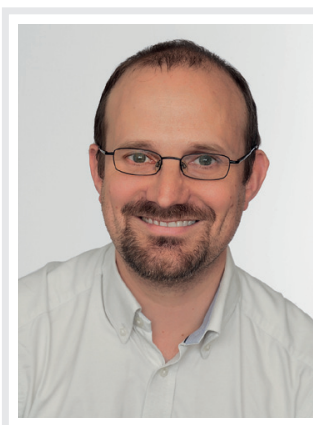

Jeremy N Burrows

Medicines for Malaria Venture, PO Box 1826, 20 route de Pré-Bois, I215 Geneva 15, Switzerland E-mail: burrowsj@mmv.org 
parasites in multi-well plates and screen in highthroughput modes based, typically, on metabolic or proliferation end-points to find compounds giving a required phenotype.

Both of these strategies have proved fruitful, with the greatest success in recent years, based on phenotypic screening. Our ability to correctly identify both validated and tractable biological targets appears limited; conversely several new, compelling but previously unforeseen targets and pathways have been identified by allowing the parasite to self-select targets or pathways for us by simply following up compounds with the most interesting phenotype and then searching for the target using reverse genetics [8]. As our understanding of which are the most relevant targets improves our ability to successfully select and prosecute target based drug discovery programs will increase.

\section{In this issue}

This issue of Future Medicinal Chemistry is dedicated to malaria drug discovery and research and brings together perspectives, reviews and preliminary communications from leading international experts in the disease with considerable experience in drug hunting.

The state of the art regarding the Plasmodium kinome and the role of these targets in control and eradication is described [9]. This is timely, given the public disclosure of thousands of submicromolar antimalarial actives of which a sizeable proportion are predicted to inhibit a kinase [10]. Meanwhile, an approach that exploits the parasite's reliance on haemoglobin degradation using the high concentration of ferrous heme as a sensor to trigger degradation of an optimized hybrid drug is explained [11]. Finally, from a target perspective, the important development and progression into healthy volunteers of the 4-pyridone (GSK932121), a mitochondrial bcl inhibitor by GlaxoSmithKline is described [12]

Two examples of maximizing the molecular diversity for phenotypic screening are reported. These are particularly pertinent given the extensive whole-cell screening that has already taken place ( $>6$ million compounds). The first is based on the innovative diversity oriented synthesis strategy that delivers novel scaffolds rich in functional and stereochemical information [13], and the second is based on an approach that exploits natural processes: using enzymes to generate diversity through biotransformations [14]. As a conclusion to asexual phenotypic screening, the identification of two series from the screening of the Biofocus Soft-Focus library is reported [15]. One of these series has subsequently led to the first preclinical candidate antimalarial from the University of Cape Town (South Africa) and the molecule, MMV390048, is progressing into preclinical development.

The need to target eradication and to discover drugs that block transmission requires an understanding of the biology of the sexual and vector stages of the parasite, the establishment of relevant assays and the delivery of new scaffolds that will fuel this next wave of eradication drug discovery. Two articles $[16,17]$ give excellent summaries of these important areas detailing the state of the art, progress and the challenges that we face as a community.

Thus the renaissance in antimalarial drug discovery is underway and progress is being made. However, we must be ever vigilant against complacency given the parasite foe that we share. Renewed and sustained efforts are therefore critical, as is a firm focus on discovering, developing and delivering new, high-quality antimalarials for the world's poor that contribute towards the goal of malaria eradication.

\section{Financial \& competing interests disclosure}

The author has no relevant affiliations or financial involvement with any organization or entity with a financial interest in or financial conflict with the subject matter or materials discussed in the manuscript. This includes employment, consultancies, honoraria, stock ownership or options, expert testimony, grants or patents received or pending, or royalties.

No writing assistance was utilized in the production of this manuscript.

5 Alonso PL, Brown G, Arevalo-Herrera M et al. A research agenda for malaria eradication: drugs. PLoS Med. 8(1), e1000406 (2011).

6 Anthony MP, Burrows JN, Duparc S, Moehrle J, Wells TN. The global pipeline of new medicines for the control and elimination of malaria. Malar. J. 11, 316 (2012).
3 Delves M, Plouffe D, Scheurer C et al. The activities of current antimalarial drugs on the life cycle stages of Plasmodium: a comparative study with human and rodent parasites. PLoS Med. 9(2), e1001169 (2012).

4 White MT, Conteh L, Cibulskis R, Ghani AC. Costs and cost-effectiveness of malaria control interventions - a systematic review. Malar. J. 10, 337 (2011). 
7 Gardner MJ, Hall N, Fung E et al. Genome sequence of the human malaria parasite Plasmodium falciparum. Nature 419(6906), 498-511 (2002).

8 Rottmann M, McNamara C, Yeung BKS et al. Spiroindolones, a potent compound class for the treatment of malaria. Science 329(5996), 1175-1180 (2010).

9 Lucet IS, Tobin A, Drewry D, Wilks AF, Doerig C. Plasmodium kinases as targets for new-generation antimalarials. Future Med. Chem. 4(18), 2295-2310 (2012).

10 Gamo FJ, Sanz LM, Vidal J et al. Thousands of chemical starting points for antimalarial lead identification. Nature 465, 305-310 (2010).

11 Mahajan SS, Gut J, Rosenthal PJ, Renslo AR. Ferrous-iron dependent delivery of therapeutic agents to the malaria parasite. Future Med. Chem. 4(18), 2241-2249 (2012).

12 Bueno JM, Herreros E, Angulo-Barturen I et al. Exploration of $4(1 \mathrm{H})$-pyridones as a novel family of potent antimalarials inhibitors of the plasmodial cytochrome bc1. Future Med. Chem. 4(18), 2311-2323 (2012).

13 Dandapani S, Comer E, Duvall JR, Munoz B. Hits, leads and drugs against malaria through diversity-oriented synthesis. Future Med. Chem. 4(18), 2279-2294 (2012).

Chigorimbo-Murefu NTL, Njoroge M, Nzila A, Louw S, Masimirembwa C, Chibale K. Biotransformation and biocatalysis: roles and applications in the discovery of antimalarials. Future Med. Chem. 4(18), 2325-2336 (2012).
15 Paquet T, Gordon R, Waterson D, Witty MJ, Chibale K. Antimalarial aminothiazoles and aminopyridines from phenotypic whole cell screening of a SoftFocus library. Future Med. Chem. 4(18), 2265-2277 (2012).

16 Delves MJ. Plasmodium cell biology should inform strategies used in the development of antimalarial transmission-blocking drugs. Future Med. Chem. 4(18), 2251-2263 (2012).

17 Lucantoni L, Avery VM. Whole-cell in vitro screening for gametocytocidal compounds. Future Med. Chem. 4(18), 2337-2359 (2012).

\section{- Website}

101 Roll Back Malaria: RBM Annual Report 2011.

www.rollbackmalaria.org 